\title{
Assessment of antibiotic susceptibility of Legionella pneumophila isolated from water systems in Poland
}

\author{
Agnieszka Sikora', Iwona Gładysz², Maria Kozioł-Montewka², Małgorzata Wójtowicz-Bobin', \\ Tomasz Stańczak', Renata Matuszewska33, Bożena Krogulska ${ }^{3}$ \\ ${ }^{1}$ Department of Medical Microbiology, Medical University of Lublin, Poland \\ 2 Pope John Paul II State School of Higher Education of Biała Podlaska, Poland \\ ${ }^{3}$ National Institute of Public Health - National Institute of Hygiene, Department of Environmental Hygiene, Warsaw, Polandd
}

Sikora A, Gładysz I, Kozioł-Montewka M, Wójtowicz-Bobin M, Stańczak T, Matuszewska R, Krogulska B. Assessment of antibiotic susceptibility of Legionella pneumophila isolated from water systems in Poland. Ann Agric Environ Med. 2017; 24(1): 66-69. doi: 10.5604/12321966.1234048

\begin{abstract}
Introduction and objective. Several studies have reported therapy failures in patients with legionnaires'disease; however, antimicrobial resistance of clinical and environmental isolates of Legionella spp. has not yet been documented. Routine susceptibility testing of Legionella spp. is not recommended because of difficulties in determining standard minimal inhibitory concentration values. The purpose of this study was to analyze the antimicrobial susceptibility of Legionella pneumophila. strains isolated from a water supply system.

Materials and method. Twenty-eight isolates of L. pneumophila (16 - L. pneumophila SG 1, 12 - L. pneumophila SG 2-14) obtained from water systems in public buildings in Poland were tested. Susceptibility testing was performed using the E-test method. The tested antibiotic were azithromycin, ciprofloxacin, and rifampicin. The medium used for the susceptibility testing was BCYE-, a special medium for Legionella cultivation.

Results. Among the tested strains, L. pneumophila was the only one resistant to azithromycin. It was a strain of $L$. pneumophila SG 2-14 isolated from the water system in a sanitorium. All isolates were found to be sensitive to ciprofloxacin and rifampicin. However, the azithromycin-resistant strain exhibited higher ciprofloxacin and rifampicin MIC $(1.5 \mu \mathrm{g} / \mathrm{ml}$, and $0.19 \mu \mathrm{g} / \mathrm{ml}$, respectively). The $\mathrm{MIC}_{50}$ for azithromycin, ciprofloxacin, and rifampicin were 0,032, 0,125, and 0,003 $\mu \mathrm{g} / \mathrm{ml}$, respectively. The $\mathrm{MIC}_{90}$ for azithromycin, ciprofloxacin, and rifampicin were $0,032,0,125$, and 0,003 $\mu \mathrm{g} / \mathrm{ml}$, respectively.

Conclusions. Azithromycin resistance was found in one strain of $L$. pneumophila SG 2-14, but the resistance mechanism is unknown and needs further study. It is possible that therapeutic failures in Legionnaires' disease may be associated with bacterial resistance which should be taken into account. The antibiotic sensitivity testing described in this study could be helpful in detecting the resistance of clinical L. pneumophila isolates. Ciprofloxacin and rifampicin have good in vitro activity against environmental L. pneumophila SG 1 and SG 2-14 in Poland.
\end{abstract}

Key words

Legionella pneumophila, susceptibility, E-test, minimum inhibitory concentration

\section{INTRODUCTION}

Legionella species are responsible for legionellosis, which may occur in two clinical forms -pneumonia (also known as legionnaires' disease) and Pontiac fever (influenza-like, mild illness). The infection is acquired through aspiration of contaminated water [1]. Legionella can proliferate in hot water distribution systems of large buildings, such as health care facilities, or in domestic water systems $[1,2]$.

Currently, more than 50 species of Legionella have been identified, some of which have been associated with human disease, for example, Legionella pneumophila, Legionella micdadei, Legionella longbeachae, Legionella dumoffii, and Legionella bozemanii [3, 4]. L. pneumophila is the most important etiological agent of legionellosis, and serogroup 1 (SG 1) accounts for more than $90 \%$ of reported human infections [5]. The mortality rate in patients with legionnaires'disease varies and depends on the clinical settings, patient population, and antimicrobial treatment

Address for correspondence: Agnieszka Sikora, Department of Medical Microbiology, Medical University of Lublin, Chodźki 1, 20-093, Lublin, Poland E-mail: agnieszka.sikora@umlub.pl

Received: 26 August 2015; accepted: 8 January 2016; first published on January 2017
[5]. The antimicrobial agents commonly used for treatment of Legionella pneumonia are macrolides and fluoroquinolones. These agents are active against intracellular Legionella spp., which can survive and proliferate in human macrophages $[5,6]$. Pontiac fever does not require antibiotics because it resolves spontaneously.

Erythromycin is the drug of choice in the treatment of Legionnaires' disease. Currently, the use erythromycin has been limited by the high incidence of side-effects, for example, phlebitis, disorders of the gastrointestinal tract, and drug interactions (e.g. immunosuppressive medications). Moreover, reversible ototoxicity was found with the use of higher doses recommended in patients with Legionnaires' disease [7].

At present, the newer macrolides (such as azithromycin) and fluoroquinolones (e.g. ciprofloxacin, levofloxacin) are recommended for the treatment of Legionnaires' disease [8]. They have a superior in vitro activity and greater intracellular penetration $[8,9]$. Also, rifampicin is used in severe cases of Legionella infection, especially in immunocompromised patients and patients with comorbidities (e.g. obstructive lung disease, or diabetes mellitus) [10]. In clinical practice, this drug is used most often in combination with other 
antimicrobial drugs because of the possibility of developing resistance $[8,9]$.

Several studies have reported therapy failures in patients with legionnaires'disease; however, the antimicrobial resistance of clinical and environmental isolates of Legionella spp. has not been yet documented $[9,11]$. Only in vitro studies showed antibiotic-resistance variants [12].

Routine susceptibility testing of Legionella spp. is not recommended because of difficulties in determining standard minimal inhibitory concentration values (MICs). This is associated with high nutritional requirements of these bacteria and inactivation of some antibiotics (for example: sulfonamide, tetracycline, polymyxin B) by charcoal which is necessary for the of Legionella species [13].

\section{OBJECTIVES}

Susceptibility testing of environmental Legionella isolates has never been published, in Poland. Therefore, the purpose of this study was to analyze the antimicrobial susceptibility of L. pneumophila strains isolated from a water supply system using the E-test.

\section{MATERIALS AND METHOD}

The E-test methods and BCYE-a agar for susceptibility testing of L. pneumophila were based on previous studies $[14,15,16]$.

Bacterial strains. Twenty-eight isolates of L. pneumophila obtained from the water systems in hospitals (15), sanatoriums (2), hotels (8) and other public buildings (3) in Poland were tested. Of these isolates, 12 strains L. pneumophila serogroup 2-14 (SG 2-14) came from own collection, and 16 strains L. pneumophila serogroup 1 (SG 1) from the National Institute of Public Health - National Institute of Hygiene (NIPH-NIH) in Warsaw, Poland. L. pneumophila were isolated during the period from January 2009 - March 2010 in Lublin Province (SG 2-14) and Mazowieckie Province (SG 1).

The isolates were stored at $-70^{\circ} \mathrm{C}$ in nutrient broth with $20 \%$ glycerol. All isolates were subcultured on buffered charcoal yeast extract agar with L-cysteine (BCYE- $\alpha$ ) which is a special medium for L. pneumophila cultivation. Plates were incubated at $37^{\circ} \mathrm{C}$ in a humidified atmosphere for $48-72$ hours. Serogroups L. pneumophila (SG 1, SG 2-14) were confirmed (after incubation) with the use of the agglutination latex test (Legionella Latex Test, Oxoid). As reference strains, L. pneumophila (Philadelphia-1) ATCC 33152 and Staphylococcus aureus ATCC 25923 (LGC Standards, United Kingdom) were used as the control [17].

The media used for susceptibility testing were BCYE- $\alpha$ for L. pneumophila and Mueller-Hinton agar for control S. aureus strain ATCC 25923. Susceptibility testing was performed using the E-test method (bioMérieux, France). The tested antibiotics were azithromycin (range 0.016-256 $\mu \mathrm{g} / \mathrm{ml}$ ), ciprofloxacin (range $0.002-32 \mu \mathrm{g} / \mathrm{ml}$ ), and rifampicin (range $0.002-32 \mu \mathrm{g} / \mathrm{ml}$ ).

The colonies of L. pneumophila (from BCYE-a medium) were suspended in sterile saline buffer (0.5 McFarland standard) and were swabbed in 3 directions on BCYE- $a$ medium. The strips of azithromycin, ciprofloxacin, and rifampicin were applied to the agar surface. The same procedure was performed for the control strains. S. aureus ATCC 25923 was inoculated in parallel onto antimicrobial agent containing Mueller-Hinton agar plates as well as BCYE- $\alpha$ plates, to determinate whether BCYE- $\alpha$ inhibited the activity of the antimicrobial agents. All procedures were undertaken according to the manufacturer's instructions (bioMérieux, France). L. pneumophila strains were culture for 48 hours at $35^{\circ} \mathrm{C}$ with an increased humidity on BCYE- $\alpha$ agar before reading the MIC values. Plates with slow-growing L. pneumophila strains were incubated for another $24 \mathrm{~h}$. S. aureus was incubated at $35^{\circ} \mathrm{C}$ for $24 \mathrm{~h}$.

MIC determination. The minimum inhibitory concentration (MIC) was defined as the lowest concentration of antimicrobial that inhibited the visible growth of a microorganisms. MICs were read at the point at which the zone of complete inhibition intersects the MIC scale. The MIC ${ }_{50}$ and MIC $_{90}$ were defined as the lowest of the antimicrobial concentrations that inhibited growth of 50 and $90 \%$ of the isolates, respectively.

Statistical analysis. MIC values between different groups (SG 1 vs. SG 2-14 L. pneumophila, hospital vs. non-hospital strains) were analyzed by Mann-Whitney U-test using STATISTICA version 10 (StatSoft, Poland). A $p$-value of $<0.05$ was considered to be statistically significant.

\section{RESULTS}

The antimicrobial susceptibility of L. pneumophila strains isolated from the water supply system of different large public buildings was analyzed. This is the first such study performed in Poland. Among the tested strains of L. pneumophila $(n=28)$, only one was resistant to azithromycin (no zone of inhibition around the E-test strip). This was a strain of L. pneumophila SG 2-14 isolated from the water system in one of the 2 sanatoria included in the study. All isolates were found sensitive to ciprofloxacin and rifampicin; however, the azithromycin-resistant strain exhibited higher ciprofloxacin and rifampicin MIC $(1.5 \mu \mathrm{g} / \mathrm{ml}$, and $0.19 \mu \mathrm{g} / \mathrm{ml}$, respectively). Further analysis during calculations are not included in the results of the strain on azithromycin resistance because it was determined the MIC value.

MICs were in the following ranges: azithromycin $0.016-0.32$ $\mu \mathrm{g} / \mathrm{ml}$, ciprofloxacin $0.004-1.5 \mu \mathrm{g} / \mathrm{ml}$, and rifampicin $0,002-$ $0.19 \mu \mathrm{g} / \mathrm{ml}$. The minimum inhibitory concentration required to inhibit the growth of $50 \%$ of L. pneumophila $\left(\mathrm{MIC}_{50}\right)$ were $0.032 \mu \mathrm{g} / \mathrm{ml}$ for azithromycin, $0.125 \mu \mathrm{g} / \mathrm{ml}$ - ciprofloxacin, and $0.003 \mu \mathrm{g} / \mathrm{ml}$ - rifampicin. The minimum inhibitory concentration required to inhibit the growth of $90 \%$ of L. pneumophila $\left(\mathrm{MIC}_{90}\right.$ ) for azithromycin, ciprofloxacin, and rifampicin were $0.032,0.125$, and $0.003 \mu \mathrm{g} / \mathrm{ml}$, respectively.

The MIC values of $L$. pneumophila SG 1 were compared with MIC values for strains of L. pneumophila SG 2-14 for all tested antimicrobial agents (Tab. 1). There was no significant difference between MIC values of strains SG 1 and SG 2-14 (azithromycin $\mathrm{p}=0.65$; ciprofloxacin $\mathrm{p}=0.42$; rifampicin $\mathrm{p}=0.07)$. Comparison of MIC values for isolates L. pneumophila from hospital versus non-hospital environments showed no significant difference in any of the tested antibiotics (azithromycin $\mathrm{p}=0.12$; ciprofloxacin $\mathrm{p}=0.27$; rifampicin $\mathrm{p}=1.0$ ). 
Table 1. Comparison of MIC values between strains of L. pneumophila SG 1 and SG 2-14

\begin{tabular}{lcccccc}
\hline \multirow{2}{*}{ Antibiotics } & \multicolumn{3}{c}{ L.pneumophila SG 1} & \multicolumn{2}{c}{ L.pneumophila SG 2-14 } \\
\cline { 2 - 7 } & Range & $\mathrm{MIC}_{50}$ & $\mathrm{MIC}_{90}$ & Range & $\mathrm{MIC}_{50}$ & $\mathrm{MIC}_{90}$ \\
\hline AZITHROMYCIN & $0.016^{\mathrm{a}}-0.32$ & 0.032 & 0.25 & $0.016-0.064$ & 0.032 & 0.047 \\
\hline CIPROFLOXACIN & $0.006-0.5$ & 0.125 & 0.19 & $0.004-1.5$ & 0.125 & 0.25 \\
\hline RIFAMPICIN & $\begin{array}{c}0.002- \\
0.006\end{array}$ & 0.003 & 0.004 & $0.002-0.19$ & 0.003 & 0.008
\end{tabular}

${ }^{a}$ without azithromycin-resistant strain (no zones of inhibition)

Reference strain S. aureus ATCC 25923 was investigated on 2 media: Mueller-Hinton (reference agar to perform susceptibility testing) and BCYE- $\alpha$ in order to determine the influence of components contained in BCYE- $\alpha$ on the MIC value. All the tested antibiotics were inhibited on BCYE- $\alpha$. The MIC values were higher (4-fold increase) in BCYE- $\alpha$, compared with MIC values on Mueller-Hinton agar (MIC value BCYE- $\alpha$ /Mueller-Hinton agar: azithromycin - 3.94; ciprofloxacin - 4; rifampicin - 4) (Tab. 2).

Table 2. MIC values for reference strains

\begin{tabular}{llccc}
\hline & & \multicolumn{3}{c}{ MIC $(\mu \mathrm{g} / \mathrm{ml})$} \\
\cline { 3 - 5 } & & $\begin{array}{c}\text { AZITHRO- } \\
\text { MYCIN }\end{array}$ & $\begin{array}{c}\text { CIPROFLO- } \\
\text { XACIN }\end{array}$ & $\begin{array}{c}\text { RIFAMPI- } \\
\text { CIN }\end{array}$ \\
\hline $\begin{array}{l}\text { ATCC 33152 } \\
\text { L. pneumophila SG 1 }\end{array}$ & BCYE-a agar & 0.023 & 0.19 & 0.004 \\
\hline $\begin{array}{l}\text { ATCC 25923 } \\
\text { S. aureus }\end{array}$ & Mueller-Hinton agar & 0.19 & 0.125 & 0.008 \\
\cline { 2 - 5 } & BCYE-a agar & 0.75 & 0.5 & 0.032 \\
\hline
\end{tabular}

The mean values of MICs for the tested antibiotics did not differ considerably from the MIC values for the reference strain (ATCC 33152 L. pneumophila SG 1).

\section{DISCUSSION}

L. pneumophila is the most common pathogenic species of the genus Legionella. responsible for communityacquired and nosocomial atypical pneumonia (hospital acquired pneumonia) in industrialized countries $[1,18]$. It is estimated that L. pneumophila causes from $<1-5 \%$ of cases of community-acquired pneumonia in adults, dependent on the geographic area [18]. This bacterium is ubiquitous in water environments worldwide. L. pneumophila can colonize tap water, cooling towers, hot water distribution systems, fountains, air conditioning systems, and medical equipment containing water [1].

The mortality of community-acquired legionnaires' disease ranges from $16-30 \%$ if untreated, or when treated with inactive antimicrobial drugs, the mortality for nosocomial legionnaires' disease can approach 50\%, given the underlying disease of the patient $[7,19]$. Early appropriate antibiotic therapy is crucial to reduce mortality in patients with legionellosis. However, several studies have shown treatment failure in patients with pneumonia caused by Legionella, which may be due to resistance in clinical isolates of L. pneumophila. This has not yet been documented [12]; therefore, the in vitro activity of antibiotic against L. pneumophila (environmental and clinical isolates) should be tested in order to monitor the possible resistant strains in different regions [12].
The intracellular location of the Legionella spp. is relevant for the efficacy of the antibiotic. The use of antibiotics in the therapy capable of achieving intracellular concentration higher than the MIC were more clinically effective than antibiotics with poor intracellular penetration [7]. Antibiotics with intracellular penetration include the macrolides, fluoroquinolones, tetracyclines, and rifampicin. These antimicrobial drugs are used in the therapy for legionnaires'disease $[7,20]$, and their effectiveness has been confirmed by clinical experience and retrospective analysis. It should be emphasized that $\beta$-lactam antibiotics are ineffective in the treatment of Legionella infections because the bacteria produce $\beta$-lactamases which inactivate this group of drugs [21].

In Poland, according to recommendations of proceedings in community-acquired respiratory tract infections, the drug of choice in Legionella infection is azithromycin. Alternatively doxycyclin, moxyfloxacin, ciprofloxacin, and clarithromycin may be used. Duration of treatment is 7-10 days, depending on the severity of the disease. Antibiotic therapy may be extended in patients with lung abscesses, endocarditis, and extrapulmonary infection [7]. Moreover, in immunocompromised patients with severe legionnaires' disease, the therapy period may be up to 21 days [7].

In the presented study, in vitro susceptibilities of L. pneumophila against commonly used antibiotics (azithromycin, ciprofloxacin, rifampicin) were performed. Antimicrobial susceptibility testing for Legionella spp. is not generally recommended for routine microbiology, because a standard method for the determination of MICs of antibiotics for Legionella spp. is not available [12].

In vitro or in vivo efficacy of antibiotics against Legionella spp. has usually been based on the determination of MIC values by micro-broth dilution, agar dilution, E-test methods, cell culture models (e. g. human monocytes), and animal models. Another method is the disc diffusion method $[7,9$, 22]. None of these methods, however, are a gold standard for the susceptibility testing of L. pneumophila [12].

The use of agar and broth dilution methods are limited because of the intracellular location of the bacteria of the genus Legionella [9]. Another limitation of the susceptibility testing methods are the specific nutritional requirements of this microorganism [14]. Legionella spp grows only on BCYE- $\alpha$ medium. This medium contain charcoal which is necessary for absorbing toxic metabolites produced during growth of Legionella [14]. In the current study, BCYE- $\alpha$ medium was used in the susceptibility test. The effect of medium components on the activity of antibiotics tested was studied. All the antibiotics tested were inhibited on BCYE- $\alpha$ in comparison with Mueller-Hinton medium. The charcoal did not cause significant changes which would affect the interpretation of the test results. Furthermore, in comparison with other tests, in this study a lower increase was obtained in the MIC values for the reference strain, and growth was constant for all study drugs $[5,13]$. In the current study, comparing the sensitivity of the reference strain with strains isolated from the water systems, there were no significant differences in the MIC values.

Some researchers report that an alternative medium used to determine the susceptibility of Legionella spp. is a charcoalfree medium - washed buffered yeast extract (WBYE), [13, $14,16,17,23]$. However, not all Legionella strains grow on this medium. In addition, a considerable decrease in growth 
has also been reported in tested strains of L. pneumophila [13]. This confirms necessity to use a conventional BCYE medium for susceptibility testing.

In the presented study, one strain was resistant to azithromycin, which also showed intermediate susceptibility to ciprofloxacin, and a much lower sensitivity to rifampicin in comparison with other tested isolates. Resistance to macrolides may be due to 3 mechanisms: ribosomal modification, efflux mechanism and drug inactivation [23]. Accurate determination of the mechanism of resistance requires further investigation.

In analyzing the results of this study (without azithromycinresistant strain), MIC values came within in the following ranges: azithromycin $-0.016-0.32 \mu \mathrm{g} / \mathrm{ml}$, ciprofloxacin $0.004-1.5 \mu \mathrm{g} / \mathrm{ml}$, and rifampicin $-0.002-0.19 \mu \mathrm{g} / \mathrm{ml}$. In the currentr study, $\mathrm{MIC}_{50}$ for azithromycin, ciprofloxacin, and rifampicin were $0.032 \mu \mathrm{g} / \mathrm{ml}, 0.125 \mu \mathrm{g} / \mathrm{ml}$, and $0,003 \mu \mathrm{g} /$ $\mathrm{ml}$, respectively. These results are similar to those obtained in previous studies, but it should be emphasized that the MIC range for antimicrobial agents may vary somewhat in different geographic regions $[12,13,25]$.

Erdogan et al. evaluated the antimicrobial susceptibility of Legionella spp. isolated from the hotel and hospital water supply system in different regions of Turkey. The researchers used a microdilution method and buffered yeast extract medium supplemented with $0.1 \%$ a-ketoglutarate (BCE- $\alpha$ ). MIC values were $0,001-0.5 \mathrm{mg} / \mathrm{L}$ for azithromycin, 0,001-0,125 mg/L for ciprofloxacin, and 0,001-0.5 mg/L for rifampicin. $\mathrm{MIC}_{50}$ for azithromycin, ciprofloxacin, and rifampicin were $0,015 \mathrm{mg} / \mathrm{L}, 0.03 \mathrm{ml} / \mathrm{L}$, and $0,001 \mathrm{mg} / \mathrm{L}$, respectively [9]. In another study evaluating the drug sensitivity of Legionella isolated in Portugal, the MIC values for ciprofloxacin and rifampicin were $0,250-1 \mu \mathrm{g} / \mathrm{ml}$ $\left(\mathrm{MIC}_{50-} 0.5 \mu \mathrm{g} / \mathrm{ml}\right)$, and $0.16-0.5 \mu \mathrm{g} / \mathrm{ml}\left(\mathrm{MIC}_{50}-0,023 \mu \mathrm{g} /\right.$ $\mathrm{ml})$, respectively [17].

When isolates from hospital environments were compared in the presented study, there was no significant difference in the MIC values. The results are similar to those obtained by other researchers [11]. Also, in the presented study there were no differences in susceptibility between serogroups of L. pneumophila (SG1 and SG 2-14).

\section{CONCLUSIONS}

Azithromycin resistance occurred in one strain of L. pneumophila SG 2-14. The resistance mechanism is unknown and needs further study. It is possible that therapeutic failures in Legionnaires' disease may be associated with bacterial resistance, which should be taken into account. The antibiotic sensitivity testing described in this study could be helpful in detecting the resistance of clinical L. pneumophila isolates. Ciprofloxacin and rifampicin have good in vitro activity against environmental L. pneumophila SG 1 and SG 2-14 in Poland.

\section{REFERNCES}

1. Phin N, Parry-Ford F, Harrison T, Stagg HR, Zhang N, Kumar K, et al. Epidemiology and clinical management of Legionnaires' disease. Lancet Infect Dis. 2014; 14(10): 1011-1021.
2. Kozioł-Montewka M, Pańczuk A, Tokarska-Rodak M, Paluch-Oleś J, Gładysz I, Sikora A, et al Current infectious threats associated with the development of civilization and progress in medicine - methods of prevention and education. Health Probl Civiliz. 2014; 8(1): 6-14.

3. Wullings BA, van der Kooij D. Occurrence and genetic diversity of uncultured Legionella spp. in drinking water treated at temperatures below $15^{\circ} \mathrm{C}$. Appl Environ Microbiol. 2006; 72(1): 157-166.

4. Hilbi H, Hoffmann C, Harrison CF. Legionella spp. outdoors: colonization, communication and persistence. Environ Microbiol Rep. 2011; 3(3): 286-296.

5. Bruin JP, Diederen BMW, Ijzerman EP, den Boer JW, Mouton JW. Correlation of MIC value and disk inhibition zone diameters in clinical Legionella pneumophila serogroup 1 isolates. Diagn Microbiol Infect Dis. 2013; 76(3): 339-342.

6. Onody C, Bernard-Matsiota P, Naściel C. Lack of resistance to erythromycin, rifampicin and ciprofloxacin in 98 clinical isolates of Legionella pneumophila. J Antimicrob Chemother. 1997; 39(6): 815-816.

7. Pedro-Botet ML, Yu VL. Treatment strategies for Legionella infection. Expert Opin Pharmacother. 2009; 10(7): 1109-1121.

8. Dedicoat M, Venkatesan P. The treatment of Legionnaires' disease. J Antimicrob Chemother 1999; 43(6): 747-752.

9. Erdogan H, Can F, Demirbilek M, Timurkaynak F, Arslan H. In vitro of antimicrobial agents against Legionella isolated from environmental water systems: first results from Turkey. Environ Monit Assess. 2010; 171(1-4): 487-491.

10. Varner TR, Bookstaver PB, Rudisill CN, Albrecht H. Role of rifampinbased combination therapy for severe community-acquired Legionella pneumophila pneumonia. Ann Pharmacother. 2011; 45(7-8): 967-976.

11. Roig J, Rello J. Legionnaires' disease: an approach to therapy. J Antimicrob Chemother. 2003; 51(5): 1119-1129.

12. Tsakris A, Alexiou-Daniel S, Souliou E, Antoniadis A. In vitro activity of antibiotics against Legionella pneumophila isolates from water systems. J Antimicrob Chemother. 1999; 44(5): 693-695.

13. Nielsen K, Bangsborg JM, Hoiby N. Susceptibility of Legionella species to five antibiotics and development to resistance by exposure to erythromycin, ciprofloxacin, and rifampicin. Diagn Microbiol Infect Dis. 2000 ; 36(1): 43-48

14. Rhomberg PR, Bale MJ, Jones RN. Application of the Etest to antimicrobial susceptibility testing of Legionella spp. Diagn Microbiol Infect Dis. 1994; 19(3): 175-178.

15. Rhomberg P, Jones RN. Evaluation of the E-test for antimicrobial susceptibility testing of Legionella pneumophila, including validation of the imipenem and sparfloxacin strips. Diagn Microbiol Infect Dis. 1994; 20(3): 159-162.

16. Pendland SL, Martin SJ, Chen C, Schreckenberger PC, Danziger LH. Comparison of charcoal- and starch-based media for testing susceptibilities of Legionella species to macrolides, azalides, and fluoroquinolones. J Clin Microbiol. 1997; 35(11): 3004-3006.

17. Marques T, Piedade J. Susceptibility testing by E-test and agar dilution of 30 strains of Legionella spp. isolated in Portugal. Clin Microbiol Infect. 1997; 3(3): 365-368.

18. Segreti J, Meyer P, Kapell K. In vitro activity of macrolides against intracellular Legionella pneumophila. Diag Microbiol Infect Dis. 1996; 25(3): 123-126.

19. Pedro-Botet L, Yu VL. Legionella: macrolides or quinolones? Clin Microbiol Infect. 2006; 12: (Suppl 3): 25-30.

20. Schrock J, Hackman BA, Plouffe JF. Susceptibility of ninety-eight clinical isolates of Legionella to macrolides and quinolones using the Etest. Diagn Microbiol Infect Dis. 1997; 28(4): 221-223.

21. Aras Z, Sayin Z. Detection of Legionella pneumophila from domestic water their antibiotic resistance profiles. J Coast Life Med. 2015; 3: 273-275.

22. Stout JE, Sens K, Mitzner S, Obman A, Yu VL. Comparative activity of quinolones, macrolides and ketolides against Legionella species using in vitro broth dilution and intracellular susceptibility testing. Int J Antimicrob Agents. 2005; 25(4): 302-307.

23. Chen SC, Paul ML, Gilbert GL. Susceptibility of Legionella species to antimicrobial agents. Pathology 1993; 25(2): 180-183.

24. Leclercq R. Mechanisms of resistance to macrolides and lincosamides: nature of the resistance elements and their clinical implications. Clin Infect Dis. 2002; 34(4): 482-492.

25. Alexandropoulou IG, Parasidis TA, Konstantinidis TG, Constantinidis TC, Panopoulou M. Antibiotic susceptibility surveillance of environmental Legionella strains: application of the E-test to bacteria isolated from hospitals in Greece. J Infect Dis Ther. 2013; 2: e103. 